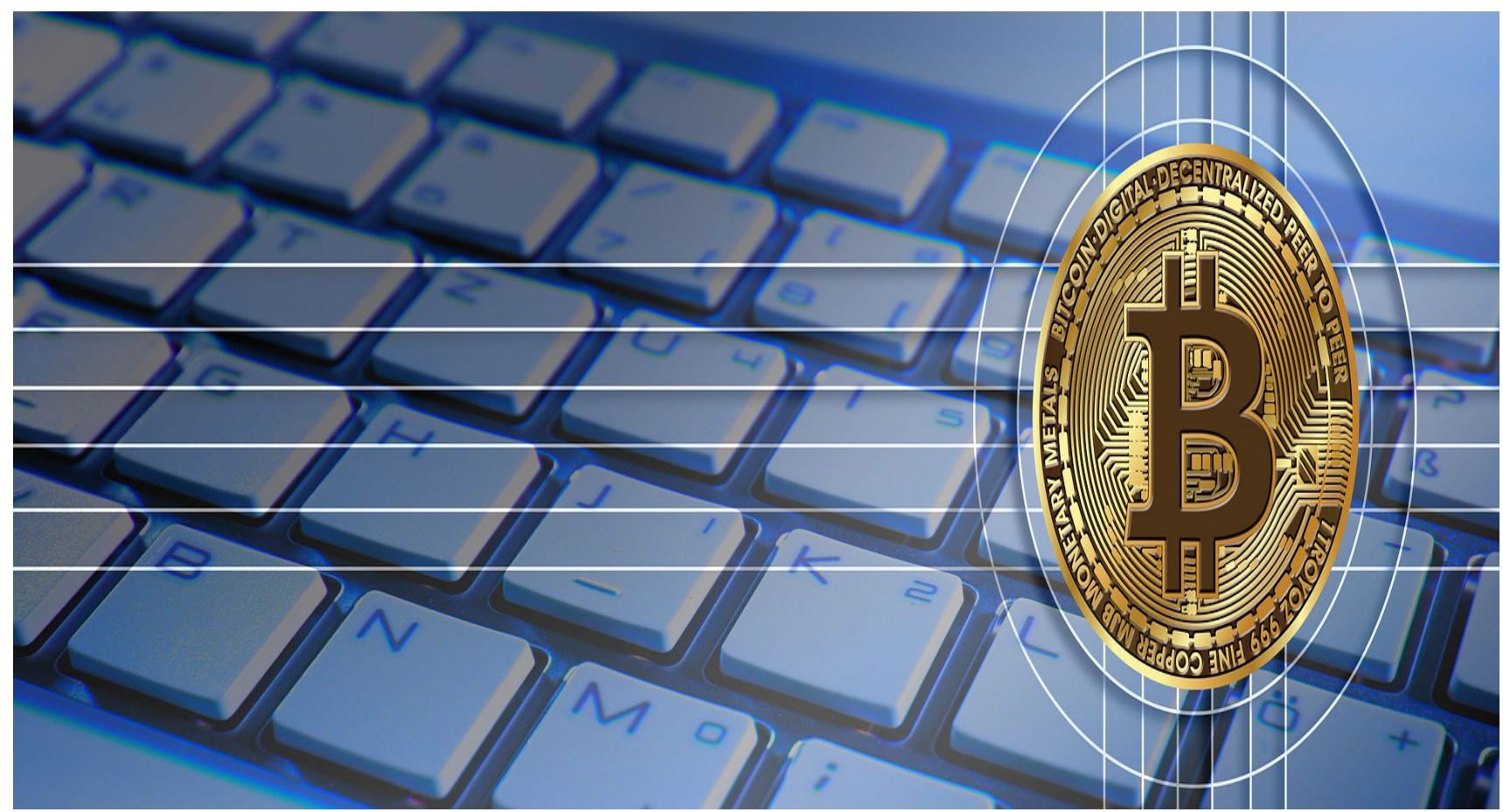

\title{
Ventajas de la facturación electrónica en empresas de Cúcuta-Norte de Santander
}

\section{Advantages of electronic invoicing in companies in Cucuta, north Santander}

\section{Blanca Liliana Carrascal-Velásquez}

Facultad de ciencias Empresariales, grupos de investigación GTN y GIPAB, bl_velasquez@fesc.edu.co ,Fundación de estudios superiores Comfanorte, Ocaña-Colombia,

\section{Johann Fernando Hoyos-Patiño}

Facultad de ciencias agrarias y del ambiente/Departamento pecuario, GI@S y GIPAB, jfhoyosp@ufpso.edu.co Universidad Francisco de Paula Santander, Ocaña-Colombia,

\section{Lilian Natalia Sayado-Velasquez}

Estudiante administracion financiera, Universidad de Santander, velasqueznati29@gmail.com,Cúcuta, Colombia

Jeimy Estefanny Sayago-Velasquez

Estudiante Bacteriología y laboratorio clínico, Universidad de Santander, sayagotefi2903@gmail.com, Cúcuta, Colombia 
Cómo citar: Carrascal-Velásquez, B.L., Hoyos-Patiño, J.F., Sayado-Velasquez, L.N., Sayago-Velasquez, J.E.

(2020). Ventajas de la facturación electrónica en empresas de Cúcuta-Norte de Santander. Reflexiones

contables (Cúcuta), 3(1), 68-81.

\section{Resumen}

En la actualidad el implementar la factura electrónica es de vital importancia, debido a que permite que el gobierno desarrolle una imposición fiscal y tributaria a través de esta medida; sin embargo en varios países de Latinoamérica, incluido Colombia estas responsabilidades de evasión han ido aumentando, es por ello que el presente artículo tiene como propósito identificar las ventajas y la importancia que representa la implementación de la facturación electrónica en las empresas de Cúcuta, así como establecer cada uno de los beneficios tanto económicos, tributarios y operativos que podrían obtener las empresas a la hora de establecer el sistema de facturación electrónica. Para ello se llevó a cabo una revisión bibliografía. Se tomaron trabajos de revistas y repositorios universitarios. Una vez revisada la bibliografía, se pudo analizar que más del $50 \%$ de la población de Cúcuta no cuenta con el conocimiento adecuado sobre la facturación electrónica, manifestando que las entidades encargadas como la DIAN no prestan el acompañamiento adecuado o manifestando la falta de recursos para contar con la herramienta digital. A pesar de los problemas presentados por la comunidad empresarial de la ciudad, se puede evidenciar que los beneficios que trae consigo la factura digital son más, puesto que permite llevar en tiempo real el comportamiento de la empresa, dar un manejo de la información de forma segura y confiable y llevar a cabo todos los procedimientos administrativos en menos tiempo, que con el uso de la factura física. Este hecho también reduce los costos de operación y personal, haciendo que la facturación electrónica se vea como una solución y una oportunidad de que la empresa pueda lograr posicionarse de manera nacional e internacional y de esta manera ofrecer una experiencia de calidad a los clientes.

Palabras clave: Comercio electrónico, economía digital, facturación electrónica, implementación, ventajas y desventajas

\section{Abstract}

Currently, the implementation of electronic invoicing is of vital importance, because it allows the government to develop a fiscal and tax imposition through this measure; however, in several Latin American countries, including Colombia, these evasion responsibilities have been increasing, which is why this article aims to identify the advantages and importance of the implementation of electronic invoicing in the companies of Cúcuta, as well as to establish each of the economic, tax and operational benefits that companies could obtain when establishing the electronic invoicing system. For this purpose, a bibliographic review was carried out. Works were taken from journals and university repositories. Once the bibliography was reviewed, it was possible to analyze that more than $50 \%$ of the population of Cúcuta does not have adequate knowledge about electronic invoicing, stating that the entities in charge, such as the DIAN, do not provide adequate support or that they lack the resources to have the digital tool. In spite of the problems presented by the business community of the city, it can be evidenced that the benefits brought by the digital invoice are more, since it allows to keep in real time the behavior of the company, to manage the information in a safe and reliable way and to carry out all the administrative procedures in less time, than with the use of the physical invoice. This fact also reduces operating and personnel costs, making electronic invoicing a solution and an opportunity for the company to position itself nationally and internationally and thus offer a quality experience to customers.

Keywords: E-commerce, digital economy, e-invoicing, implementation, advantages and disadvantages 


\section{0}

\section{Introducción}

En la actualidad la implementación de la facturación electrónica es de suma importancia, debido a que los gobiernos han desarrollado una imposición sobre esta medida, puesto que facilita el control de evasión fiscal y la gestión tributaria. Sin embargo en varios países de Latinoamérica, incluido Colombia, estas responsabilidades de evasión han ido aumentando, permitiendo visibilizar la importancia del uso de esta medida, la factura digital (Roncallo, 2019).

Es por esto que como lo menciona (Eladio \& Ballesteros, 2012), a través de la historia la aplicación de la tecnología ha permitido que diferentes tipos de empresas generen ventajas competitivas a través del uso del comercio electrónico, generando un incremento en las expectativas de los clientes y en cómo se realiza el tratamiento de la información y la comunicación. Por otro lado, de acuerdo con (Arévalo \& Ortega, 2019) la evolución de la economía ha permitido que los avances en la tecnología busquen optimizar los recursos, generar eficiencia en cada proceso, y una mayor eficacia en quienes intervienen, desarrollando una relación comercial impersonal al momento de usar las plataformas digitales, en transacciones como la compra y vienes de productos o servicios, asentando que la factura electrónica sea el principal soporte en el proceso.

Todas aquellas empresas que entren al proceso de la facturación electrónica deberán realizar modificaciones sensibles en sus servicios técnicos, debido a que todos los procesos cambiarían de acuerdo a las nuevas metodologías. Para esto, en primer lugar se deben identificar los diferentes pro y contra de la implementación de la facturación electrónica, con el objetivo de determinar un proceso adecuado y personalizado de acuerdo a cada empresa y por último desarrollar una serie de capacitaciones, donde los empleados de la empresa cuenten con toda la información necesaria y de mayor calidad (Beltrán, 2018).

Es por esto que como es mencionado por (Gomez \& Sanchez, 2018) dentro de la economía mundial, se da como prioridad la necesidad de estandarizar los diferentes procesos y/o mecanismos que generen herramientas para un mejor acceso a la información y para llegar a esto, se debe tener en cuenta la aplicabilidad y el mejor entendimiento de la normativa colombiana referente al tema. De allí la necesidad de generar una serie de ventajas significativas, relacionándolas con la realidad del país, sobre la forma eficiente y sencilla de la implementación de la factura electrónica en la ciudad. Para esto se tendrá en cuenta diferentes sectores empresariales, los cuales han formado y forman parte del patrimonio cultural y económico de la región.

Sin embargo la población colombiana, específicamente en la ciudad de Cúcuta ha permitido visualizar una gran resistencia a la hora de cambiar la factura convencional a la facturación electrónica, ya que la cultura tributaria evidenciada en cada uno de los contribuyente es deficiente, lo que permite evidenciar que existen antecedentes de corrupción, haciendo que las personas que en su labor comercial asocian esta modalidad electrónica, como una forma en que el estado ejerce vigilancia y control sin medida, donde el único objetivo de este es captar una mayor cantidad de recursos a través del pago del IVA de forma legal y a su vez sean desviados, desconociendo las ventajas que trae el implementar este nuevo sistema en las actividades económicas de la región (Díaz, Coba, \& Bombón, 2016).

De acuerdo con lo anteriormente mencionado, el presente artículo tiene como propósito identificar las ventajas y la importancia que representa la implementación de la facturación electrónica en las empresas de Cúcuta, así como establecer cada uno de los beneficios 
tanto económicos, tributarios y operativos que podrían obtener las empresas de a la hora de establecer el sistema de facturación electrónica como lo manifiestan Rivera et al (2019).

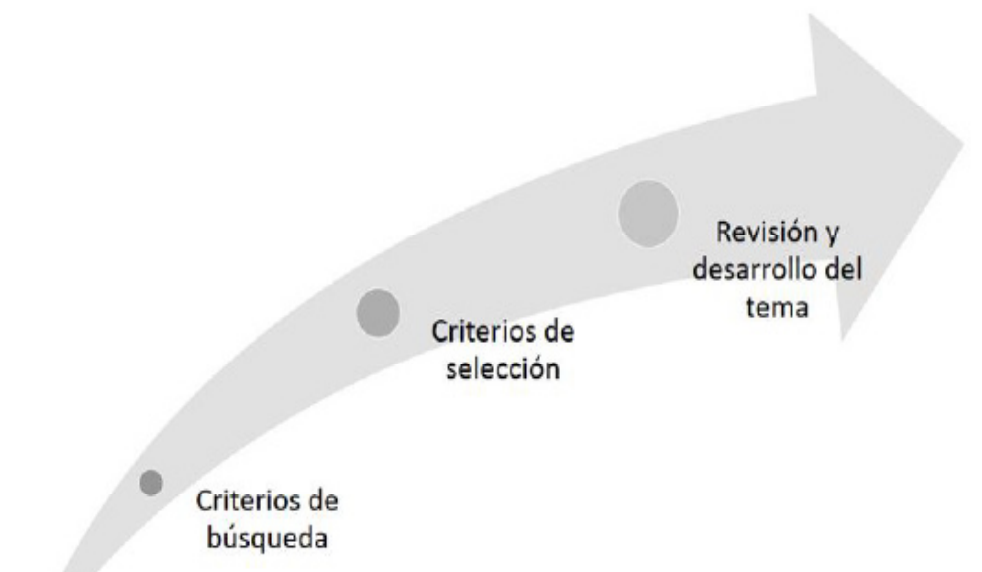

Figura 1. Metodología para la elaboración del artículo.

Fuente: Autores

En la figura número 1 se aprecia las etapas que se llevaron a cabo para el desarrollo del artículo. En una primera fase, se procedió a una búsqueda exhaustiva bibliográfica. Los artículos relacionados con la facturación electrónica, sus ventajas y beneficios, los cuales fueron temas primordiales para la revisión. Se seleccionaron diferentes artículos de cualquier parte del mundo para una revisión completa, usando bases de datos y repositorios universitarios. También, para la recopilación de los artículos se tuvieron en cuenta las palabras clave elegidas, las cuales son: Facturación electrónica, ventajas y desventajas, implementación, comercio electrónico y economía digital.

Después de esta primera fase se recurrió a la selección de los documentos usando como rango de fechas el año 2010 al 2020, donde se utilizaron los criterios de búsqueda con palabras clave mencionadas anteriormente. En una segunda fase, también se utilizó como criterio de selección aquellas revistas indexadas y repositorios universitarios, que permitieran encontrar con mayor facilidad el tema a tratar de este artículo. Por consiguiente, algunas de éstas revistas y universidades fueron: Ciencia UNEMI, Económicas Cuc, Institución Universitaria ESUMER, Universidad Libre de Colombia, Universidad de Guayaquil, Universidad San Ignacio de Loyola, entre otras.

Como fase final, se llevó a cabo la escritura y composición del presente trabajo con la información correspondiente al tema escogido. Para ello, los artículos y trabajos de investigación que fueron seleccionados se organizaron en una matriz de extracción, la cual permitió identificar las ventajas que representa la implementación de la facturación electrónica en las empresas de Cúcuta.

\section{Resultados y análisis}

\section{Comportamiento de las Empresas en Cúcuta sobre la Facturación Electrónica}

De acuerdo con lo estudios realizados, deja en evidencia a los empresarios de la ciudad de Cúcuta, debido que a pesar de reconocer las facilidades que pueden contraer al implementar la factura electrónica, estos optan por no realizarla puesto que el suministro de la factura digital y a su vez la declaración de la 


\section{2}

misma a través de los diferentes impuestos, genera un cierto fraude y evasión fiscal, acciones que muestran la realidad por la que pasan las diferentes entidades en cuanto a la gestión de administración y control fiscal (Morantes \& Carvajal, 2018).

Por otro lado, otras de las dificultades que se presentan para la implementación de la facturación electrónica en la ciudad de Cúcuta, es el hecho del desconocimiento de la misma o la falta de capacitación y acompañamiento a los empresarios de la región para que se asegure ese proceso y mejorar sus procesos operacionales y contables. Como se puede ver en la Figura 2, a pesar de la evidencia del conocimiento sobre la factura electrónica en más del $50 \%$ de la población, se presenta un porcentaje considerable de empresarios que no cuentan con el total conocimiento en cuanto a este proceso, ya que como se puede observar un $38 \%$ no tiene claridad sobre las estrategias para la implementación de la factura electrónica, sus etapas y su marco legal; un $63 \%$ no cuenta con el conocimiento sobre los modelos de emisión y recepción del documento digital; y por último $25 \%$ no tiene conocimiento sobre la firma digital, punto importante al momento de presentar un documento de factura electrónica (González, Becerra, \& Monroy, 2019).
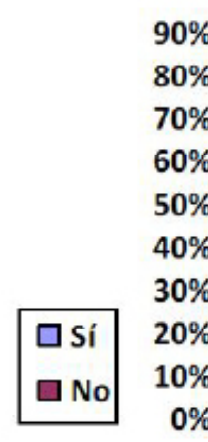

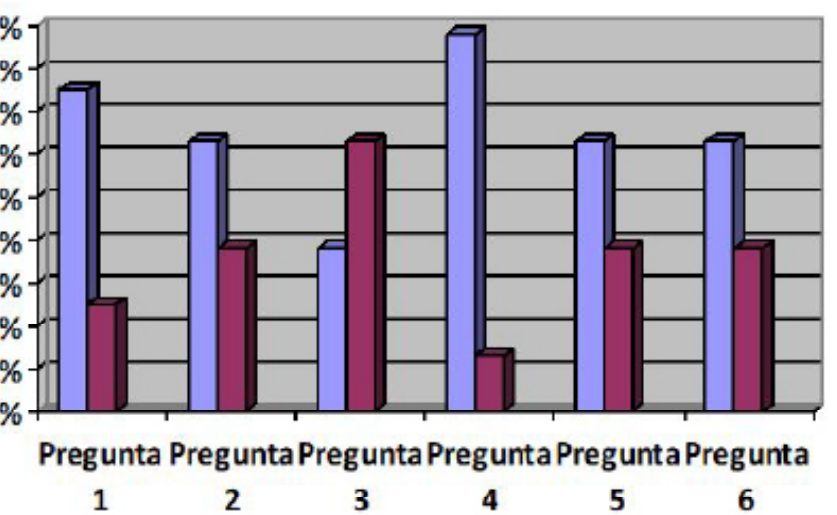

Preguntas:

1. ¿Conoce qué es la firma digital?

2. ¿Tiene claridad sobre estrategias para implementar la factura electrónica?

3. ¿Tiene conocimiento acerca de los modelos de emisión y recepción de la factura electrónica?

4. ¿Sabe usted acerca de proveedores tecnológicos que le puedan prestar el

Figura 2. Representación porcentual del conocimiento sobre la facturación electrónica de los empresarios de la ciudad de Cúcuta Fuente: (González et al., 2019)

Por otra parte a pesar del conocimiento que han adquirido los empresarios de Cúcuta sobre la factura electrónica, se evidencia en la Figura 3 que solo el $50 \%$ de ellos han implementado la factura digital en sus empresas, un $25 \%$ no ha iniciado el proceso y un último $25 \%$ que está en proceso de implementación. Esto al igual que la información presentada en la Figura 2, evidencia la falta de acompañamiento por parte de las entidades encargadas, para que todas las empresas logren iniciar el cambio de la factura física a la digital. Otra de las limitantes que se evidencia, es la falta de recursos financieros los cuales son requeridos para la estructura tecnológica y digital en todo este proceso. Por último, en Cúcuta aún no se evidencia una cultura hacia la innovación, la tecnología y la gestión del cambio, elementos que son importantes en la gestión de calidad empresarial y una mejor competitividad en la actualidad (González et al., 2019).

\section{En proceso}
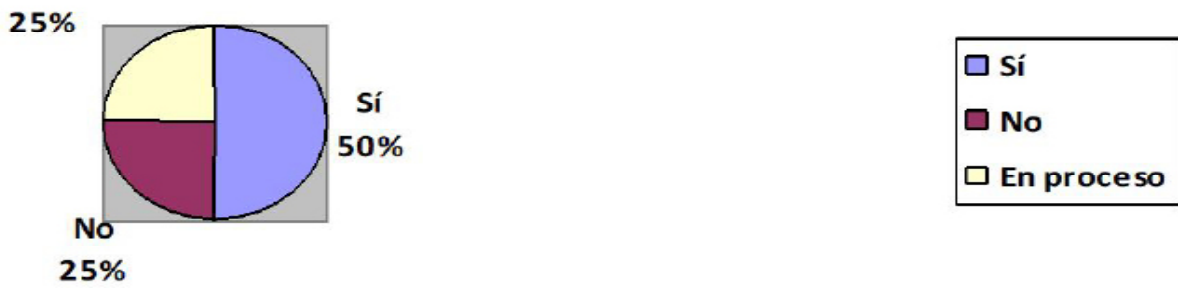

Figura 3. Implementación de la facturación electrónica 
De acuerdo a lo anteriormente mencionado, a continuación se presentan las diferentes importancias y ventajas que trae consigo la implementación de la facturación electrónica para las empresas de la ciudad de Cúcuta, al igual beneficios tanto económicos, tributarios y operativos que conlleva el llevar a cabo este proceso de digitalización en las facturas.

\section{Importancia de la Facturación Electrónica en las Empresas}

En el transcurrir del tiempo, diferentes países ha desarrollado su proceso de implementación de la facturación electrónica a paso lento, y Colombia no se aleja de esa realidad, específicamente en la ciudad de Cúcuta donde como se pudo evidenciar anteriormente, no se da prioridad por la capacitación y apoyo a los empresarios, lo que hace que la cultura tecnológica y el avance se vea pausado.

Sin embargo para el país existe el reto en que los empresarios, ya sean pequeños, medianos o grandes, logren adaptarse e implementar este nuevo sistema el cual traerá consigo diferentes beneficios. Sin embargo la Dirección de Impuestos y Aduanas Nacionales - DIAN, debe ser la más interesada en que este proceso sea llevado a cabo con total cabalidad, puesto que para este ente nacional el hecho que se presente este tipo de facturación, permite que las empresas entreguen en tiempo real todo tipo de información en las diferentes transacciones realizadas, logrando un impacto significativo en el control fiscal a la empresa emisora. (Pinzon, 2019).

Tabla 1. Impacto operativo a la hora de implementar la factura electrónica por medio de un proveedor tecnológico o de forma directa.

\begin{tabular}{|c|c|c|}
\hline PROCESO & $\begin{array}{l}\text { PROVEEDORES } \\
\text { TECNOLÓGICOS }\end{array}$ & $\begin{array}{l}\text { FACTURACIÓN } \\
\text { DIRECTA }\end{array}$ \\
\hline $\begin{array}{l}\text { Tiempos entrada en } \\
\text { producción }\end{array}$ & Inferior & Superior \\
\hline Esfuerzo & Inferior & Superior \\
\hline $\begin{array}{l}\text { Inversión en } \\
\text { infraestructura, personas } \\
\text { y certificados }\end{array}$ & Inferior & Superior \\
\hline Espacio físico & Inferior & Medio \\
\hline Mejor servicio al cliente & Superior & Medio \\
\hline Soporte técnico & Medio & Superior \\
\hline $\begin{array}{l}\text { Ajuste de desarrollo por } \\
\text { cambio de ley }\end{array}$ & Inferior & Superior \\
\hline
\end{tabular}




\section{4}

Por otro lado, el implementar este sistema permitiráque losempresarios puedanoptimizar diferentes procesos administrativos, contables y gerenciales, teniendo en cuenta si se opta por los diferentes proveedores tecnológicos y si se hace de manera directa (Scatolon, 2014). En la Tabla 1, se logra observar el impacto en los diferentes procesos operativos en caso de emitir la facturación electrónica por medio de un proveedor o si de hace de forma directa. Allí se evidencia que el hecho de generar el documento digital de forma directa presenta un impacto más alto, que si se realizar por medio de un tercer, ya que estos cuentan con la infraestructura, la capacitación y la tecnología necesaria para llevar a cabo este proceso.

Sinembargo, el hecho de realizareste procesoya sea de forma directa o por medio del proveedor, se obtendrán diferentes beneficios, los cuales podrían ir desde el tiempo de generación y preparación de facturas, la disminución en los errores de tipo administrativo, minimización en gastos de tipo operativos, y una considerable reducción y/o disminución en los gastos de las emisiones y recepciones de estos documentos digitales; significando a simple vista una mejora en la gestión de calidad empresarial y una mejora en la competitividad en relación a aquellas empresas que no han realizado la implementación de la factura electrónica (Beltrán, 2018).

\section{Ventajas de la Implementación de la Facturación Electrónica en Empresas de Cúcuta}

De acuerdo con el Departamento de Impuestos y Aduanas Nacionales - DIAN, la factura electrónica es un mecanismo que permite el soporte en las transacciones en cuanto a la venta de bienes y/o servicios, la cual es manipulada totalmente de manera digital, limitando el uso del papel tradicional, pero con los mismos propósitos impositivos y legales vigentes (Quintero \& Duran, 2019). Es por esto que como se evidencia en la Tabla 2, son más los beneficios o las ventajas que tiene el uso de la factura digital, contra la tradicional factura física o de papel. 
Tabla 2. Ventaja en la implementación de la Factura Electrónica en comparación con la Factura Tradicional.

\begin{tabular}{lcc}
\hline CARACTERISTICAS & $\begin{array}{c}\text { FACTURA } \\
\text { ELECTRÓNICA }\end{array}$ & $\begin{array}{c}\text { FACTURA } \\
\text { TRADICIONAL }\end{array}$ \\
\hline $\begin{array}{l}\text { Se elabora en un formato } \\
\text { estándar definido por la } \\
\text { DIAN }\end{array}$ & Ventaja & Desventaja \\
$\begin{array}{l}\text { Se entregan a los clientes } \\
\text { en tiempo real vía internet }\end{array}$ & Ventaja & Desventaja \\
$\begin{array}{l}\text { Genera ahorros en papel, } \\
\text { distribución y } \\
\text { conservación. }\end{array}$ & Ventaja & Desventaja \\
$\begin{array}{l}\text { Son confiables porque } \\
\text { vienen firmadas } \\
\text { digitalmente }\end{array}$ & Ventaja & Desventaja \\
$\begin{array}{l}\text { Pueden ser fácilmente } \\
\text { incorporadas en procesos } \\
\text { automáticos a los sistemas } \\
\text { contables de los clientes }\end{array}$ & Ventaja & \\
$\begin{array}{l}\text { Se consultan fácilmente } \\
\text { con un computador o } \\
\text { dispositivo electrónico }\end{array}$ & Dentaja & \\
$\begin{array}{l}\text { Se minimiza el riesgo de } \\
\text { perdida }\end{array}$ & & Desventaja \\
$\begin{array}{l}\text { Son amigables con el } \\
\text { planeta }\end{array}$ & Ventaja & \\
$\begin{array}{l}\text { Facilitan el } \\
\text { relacionamiento con los } \\
\text { clientes }\end{array}$ & Ventaja & Desventaja \\
\hline
\end{tabular}

Fuente: (DIAN, n.d.).

Además de los beneficios presentados en la Tabla 2, el hecho de manejar y/o implementar la factura digital trae consigo mejoras en los procesos internos de las empresas. Según estudios realizados, esta implementación podría hacer que las organizaciones reduzcan hasta un $80 \%$ en los costos, a comparación del proceso tradicional. Por otro lado también trae beneficios en los actores involucrados en todos los procesos, como lo son clienteproveedor, ya que en primer lugar estarían viendo una considerable disminución en el consumo de papel, disminuyendo los impactos ambientales detrás de ese proceso, así como la minimización en el uso de diferentes equipos digitales o tecnológicos como impresoras y tintes. Por último, también se reducen gastos en personal y transporte de entrega de los diferentes documentos físicos relacionados con la facturación (León, 2018).

Una de las ventajas o beneficio más significativo que representa el uso de la factura digital, es el hecho de la seguridad, puesto que en este tipo de proceso se representa el uso de la firma digital, así como los certificados electrónicos, lo cual permite crear una cultura totalmente diferente y de seguridad tecnológica en la empresa, puesto que si se sigue con el uso de la factura de papel, al momento de tener que ser transportada al destinatario, podrían ocurrir ciertas situaciones las cuales podrían 


\section{6}

convertirse en inconvenientes para el emisor o receptor, e incluso poder perder la vente o el cliente (Pérez, 2019).

La facturación electrónica en la actualidad permite que las diferentes empresas $\mathrm{u}$ organizaciones generen un tipo de soporte digital, en diferentes formatos de tipo electrónico, lo que facilita los métodos relacionados con la producción, almacenamiento, tratamiento y trasmisión de la información, llegando al ámbito tributario nacional, permitiendo que se dé una mayor eficiencia en la gestión de la información entre los facturadores (vendedores), compradores y el Departamento de Impuestos y Aduanas Nacionales - DIAN (Arévalo \& Ortega, 2019).

Finalmente la factura de tipo digital, permite a las diferentes empresas la posibilidad de estar dentro del rango de las tendencias a nivel empresarial y a nivel global, lo que genera una mejora en la calidad y a su vez en la oportunidad de participar en mercados internacionales, esto último con ceros limitaciones en cuanto al manejo de la información y la transparencia que caracteriza cada una de las transacciones comerciales (Alfaro, 2018).

\section{Ventajas Tributarias a la hora de Implementar la Facturación Electrónica}

Tributariamente la factura digital o electrónica tiene diferentes beneficios los cuales permiten que la empresa pueda realizar procedimientos de manera inmediata a comparación del uso de la factura física. En primer lugar, como fue mencionado anteriormente, la factura electrónica debe ser manejada o empleada de forma directa por personal de la misma organización, o por un tercero el cual tenga las competencias necesarias sobre el tema, lo que permita que los procesos tengan total garantía en cuanto a seguridad, autenticidad e integridad en cuanto a la facturación digital, las distintas notas créditos desde el momento en que fue expedida y el tiempo que dure su preservación. Todo esto siempre y cuando la persona que adquiera los servicios o productos ofrecidos por la empresa, acepte recibir este modelo de factura (Eladio \& Ballesteros, 2012).

Una vez obtenido el aval por parte de la persona adquiriente, para la operación contable de la empresa se le es más ágil todo el proceso de facturación y del tratamiento de la información, ya que como se puede observar en la Tabla 3, los procesos realizados a través de la factura física son más demorados que los realizados de manera digital, específicamente el traslado de la información en formato, puesto que esta actividad requiere de personal extra y tiempo extra para transferir toda la información suministrada en las facturas y en algunas ocasiones llegar a errores humanos por caligrafía a la hora de redactar los elementos que trae la factura física. 
Tabla 3. Comparación en la duración de los procesos en Factura Física y Factura Digital.

PROCEDIMIENTO

\section{DURACIÓN EN HORAS DEL PROCEDIMIENTO}

Factura Física

Revisión de Información

Extracción de la Información

Traslado de la Información Formato

Validación de la Información

Envío de Información a la

DIAN
$5-10$

$0-5$

40

5

$5-10$

\section{Factura Digital}

5

$0-5$

10

5

5

Fuente: (Eladio \& Ballesteros, 2012)

Como adición a lo anteriormente comentado como beneficio tributario, la facturación electrónica permite visualizar otro tipo de ventajas en el ámbito tributario de acuerdo a la implementación de este tipo de documento como son los siguientes (Arévalo \& Ortega, 2019):

- Cuenta con igual validez como la factura física o de papel.

- Genera la oportunidad de realizar la facturación a tanto a clientes ocasionales, como constantes con igualdad de condiciones.

- Permite que la fiscalización de todos los procesos sea llevada a cabo de forma inmediata, sencilla y con un costo reducido.

- Desarrolla la ocasión que los compromisos y obligaciones fiscales sean manejadas de forma simplificada.

- Permite que los procesos de auditoria tanto interna, como externa tengan los accesos de manera rápida y eficaz.

\section{Ventajas Económicas a la hora de Implementar la Facturación Electrónica}

De acuerdo a cada uno de los procesos que se llevan a cabo dentro de las empresas, todos conllevan un esfuerzo económico y más en este caso cuando se implementa la facturación electrónica, puesto que este procedimiento requiere de personal calificado y material tecnológico con el cual se pueda desarrollar la infraestructura digital que conllevará a la forma digital de facturar (Doliet, 2016).

Existen una serie de costos que ya se encuentran asociados a la implementación de la facturación electrónica, como lo son el diseño, el desarrollo y la capacitación. En primer lugar, el diseño es de suma importancia puesto que es el punto de partida en todo proceso de facturación electrónica, debido que a partir de este proceso se planea el sistema que se empleará en la facturación. Posteriormente el desarrollo permite estructurar el software y las interfaces que tendrá el aplicativo, de forma que se pueda tomar el mayor provecho al sistema. Por último, la capacitación tiene como objetivo formar al personal que se encargará 


\section{8}

de manejar el sistema, asumiendo todo tipo de responsabilidad para desarrollar la actividad de la facturación (Montaño, 2019).

Sin embargo, a pesar de los gastos que se mencionan anteriormente, el proceso de facturación electrónica a corto, mediano y largo plazo permite reducir de manera considerable todos los procesos operativos referentes a la contabilidad. Como se puede observar en la Figura 4, todo el proceso digital tanto en el emisor, como el receptor, permite que se reduzca de manera económica y en tiempo diferentes procedimientos en cuanto a la facturación, mejorando la calidad en la atención al cliente y la competencia con las demás empresas (Eladio \& Ballesteros, 2012).

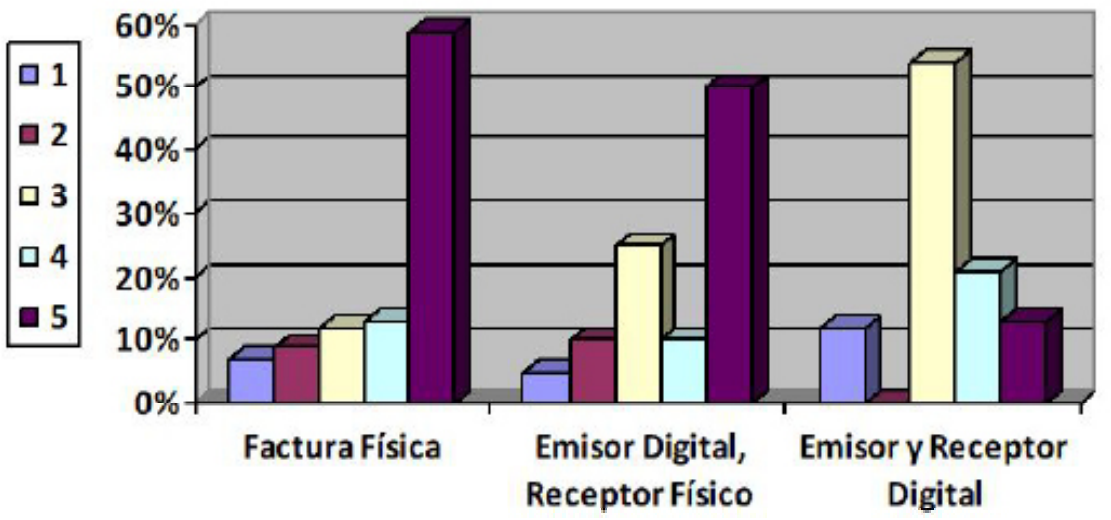
Consideraciones
1. Impresión
2. Perdida papeles
valorados
3. Procesamiento
4. Almacenamiento
5. Despacho físico

Figura 4. Porcentaje de costos en el uso de factura física y electrónica.

Fuente: (Eladio \& Ballesteros, 2012)

\section{Ventajas Operativas a la hora de 2. Genera mayor confianza y rapidez en los Implementar la Facturación Electrónica procesos de información}

Cuando la empresa desea desarrollar la facturación electrónica dentro de su actualización empresaria, como se mencionó anteriormente, permite que esta pueda lograr ingresar a nuevos mercados, tanto nacionales como internacionales, puesto que mejorar sus servicios, ampliando su gestión de calidad y permitiéndose ser más competitivo frente a las demás empresas, Mora et al., (2019). Es por esto que el hecho de presentar la factura de manera digital, permite visibilizar diferentes beneficios de tipo operativo, los cuales generan ahorros en tiempo del procedimiento comercial (compra y venta) y costos; a continuación se mencionan los beneficios más representativos (Medina V \& Rodríguez P, 2018).

1. Disminución en la falsificación de documentos
3. Corrección inmediata en caso de presentarse errores en la facturación

\section{Recepción y envío inmediato de las facturas}

5. Mayor eficiencia en el recurso humano disponible

6. Permite mejorar la imagen empresarial y/o corporativa ante los clientes

7. Tramites rápidos en los flujos financieros

8. Ausencia de pedidos y errores formales

Cabe resaltar que a pesar de observar los múltiples beneficios que se obtienen al implementar la facturación electrónica, en la actualidad existe aún el rechazo hacia este tipo de actividades, puesto que no 
existe ese acompañamiento por parte de los entes encargados. Es por esto que se deben establecer estrategias, las cuales identifiquen de manera acertada aquellas empresas que están en voluntad de hacer el cambio digital y realizar el asesoramiento para que tanto los empresarios, como la DIAN puedan llevar todos los procesos de manera organizada y actualizada (Díaz et al., 2016).

\section{Conclusiones}

En Colombia, específicamente en la ciudad de Cúcuta, las diferentes empresas no cuentan con el suficiente conocimiento en cuando al tema de la facturación electrónica y a su vez los beneficios que trae consigo su uso, lo que generaría un mejor servicio a los clientes y por otro lado la agilización en diferentes procesos tanto de tipo operativos, administrativos y contables. Por otro lado, este sistema si se empleara de la forma correcta, brindaría que la información contenida en este tipo de documento sea totalmente verás, oportuna y confiable, debido a que el sistema permite que todo se de en tiempo real y monitoreado por la Dirección de Impuestos y Aduanas Nacionales - DIAN.

Sin embargo, tanto la DIAN como las empresas interesadas, deben buscar la manera en que se consiga esa alianza estrategia, la cual permita que los empresarios puedan optar por esta medida, obteniendo todas las herramientas necesarias y la capacitación por parte del personal calificado, mejorando los estándares de calidad de las organizaciones y a su vez brindar la mejor atención a los clientes.

Cabe resaltar que cada una de las empresas, o sus representantes legales deben dar un gran esfuerzo, ya que incursionar en este tipo de tecnologías conlleva a una inversión tanto en capital, como en marketing, ya que de esta forma se debe dar a conocimiento público cada uno de los servicios o bienes que ofrecen las empresas, los cuales son totalmente respaldados por la factura electrónica, y detrás de ella los requisitos que son validados de manera fiscal y tributaria.

A pesar de todos los problemas que se evidencian, se puede observar que los beneficios son más y que la implementación de la factura digital podría mejorar la economía, los procesos operativos y los procesos tributarios de las empresas en la ciudad de Cúcuta que quieran iniciar este camino de la digitalización. Por otro lado, se podrá ofrecer una mejor calidad en su servicio y en sus productos, ya que al usar este mecanismo se genera una mayor confianza en el cliente, puesto que pueden estar totalmente seguros que toda la información allí contenida cuenta con total confidencialidad y un adecuado tratamiento.

\section{Referencias}

Alfaro, J. D. P. (2018). Implantación De Sistema De Facturación Electrónica En La Empresa World Duty Free Group Perú S.A.C. Universidad San Ignacio de Loyola. Retrieved from http:// repositorio.usil.edu.pe/handle/USIL/3933

Arévalo, A., \& Ortega, M. E. (2019). Beneficios de la implementación de la facturación electrónica en las pequeñas, medianas empresas y personas naturales. Universidad Cooperativa de Colombia. https://doi.org/.1037//0033-2909. I26.1.78

Beltrán, A. F. (2018). Implementación facturación electrónica en Colombia. Universidad Católica de Colombia. Universidad Católica de Colombia. https://doi.org/10.18041/2382-3240/ saber.2010v5n1.2536

Carrascal, B. L. V., Patiño, J. F. H., Villamizar, D. A. H., Velasquez, L. N. S., Velásquez, J. E. S., \& Yuncosa, J. A. V. (2020). (DIE)MODELO PARA EL DISEÑO DE IDEAS DE 


\section{0}

EMPRENDIMIENTO. FAGROPEC-Facultad de Ciencias Agropecuarias, 12(1), 52-64. Obtenido de: https://www.uniamazonia.edu.co/ revistas/index.php/fagropec/article/view/1816

DIAN. (n.d.). Beneficios de la Factura Electrónica. Retrieved October 12, 2020, from https://www. dian.gov.co/impuestos/factura-electronica/ factura-electronica/Paginas/beneficios.aspx

Díaz, J., Coba, E., \& Bombón, A. (2016). Facturación electrónica versus facturación clásica. Un estudio en el comportamiento financiero mediante estudios de casos. Ciencia UNEMI, 9, 63-72. Retrieved from http://ojs. unemi.edu.ec/index.php/cienciaunemi/article/ view/305/267

Doliet, C. A. (2016). Análisis del sistema de facturación electronica y su apliación en las empresas cartoneras en Guayaquil. Universidad de Guayaquil.

Eladio, F., \& Ballesteros, M. (2012). Ventajas de la implementación de la factura electrónica en Colombia. Universidad Piloto de Colombia.

Gomez, N., \& Sanchez, D. A. (2018). El impacto que tendrá la facturación electrónica en la empresa TELE VVD S.A.S. Universidad Agustina.

González, J., Becerra, D., \& Monroy, R. (2019). Estrategias para la implementación de la facturación electrónica para las mipymes en Cúcuta, Colombia. Universidad Libre de Colombia, 1-20. Retrieved from https:// repository.unilibre.edu.co/handle/10901/17891

León, M. Y. (2018). Implementación del proceso de Facturación Electrónica en la Corporación Brinks de Colombia S.A. Corporación Univesitaria Minuto de Dios.

Medina V, Ma. A., \& Rodríguez P, D. (2018). La Facturación Electrónica y su
Contribución al Medio Ambiente Seminario. Universidad Cooperativa de Colombia. Retrieved from https://repository.ucc.edu. co/bitstream/20.500.12494/6822/1/2018 facturacion_electronica_contribucion.pdf

Montaño, I. (2019). Beneficios que genera la implementación de facturación electrónica en la dirección de impuestos y aduanas nacionales (DIAN). Universidad Cooperativa de Colombia.

Mora-Villamizar, D. A., Morales-Pérez, K. T., Barrientos-Monsalve, E. J., \& VelázquezCarrascal, B. L. (2019). Análisis de la competitividad entre las empresas los olivos y la esperanza en Cúcuta, Norte de Santander-según las cinco fuerzas de Michael Porter. Revista CONVICCIONES, 6(11), 69-75. Obtenido de: https://www.fesc.edu.co/Revistas/OJS/index. $\mathrm{php} /$ convicciones/article/view/418

Morantes, S., \& Carvajal, M. V. (2018). Implementación de la factura electrónica en Colombia a partir del Decreto 2242 de 2015, en las MIPYMES del barrio La Merced de la ciudad de Cúcuta. Universidad Libre de Colombia. Universidad Libre de Colombia. https://oi. org/10.18041/2382-3240/saber.2010v5n1.2536

Pérez, J. L. (2019). Implementación de la facutra electrónica y sus beneficios en el consorcio de frutas Lambayeque SAC - 2017. Universidad Señor de Sipán.

Pinzon, A. (2019). Implementación, evolución e impacto de la facturación electrónica en Colombia. Universidad Militar Nueva Granada. Retrieved from https://repository.unimilitar. edu.co/bitstream/handle/10654/34978/ PinzonCastro Anyela Natalya 2020 . pdf?sequence $=1$

Quintero, L. P., \& Duran, Ma. L. (2019). Impacto de la factura electrónica en el sector comercial de Ocaña, Norte de Santander, 
en el período 2019. Retrieved from http:// repositorio.ufpso.edu.co:8080/dspaceufpso/ bitstream/123456789/2290/1/32100.pdf

Rivera-Lema, A. F., Urbina-Ramírez, N. G., SilvaPérez, I. E., Barrientos-Monsalve, E. J., \& Velázquez-Carrascal, B. L. (2019). Analysis of the economic revenue management of the graphic designer in Colombia. Revista CONVICCIONES, 6(11), 23-29. Obtenido de: https://www.fesc.edu.co/Revistas/OJS/index. php/convicciones/article/view/383

Roncallo, R. (2019). Facturación electrónica en Colombia: una reflexión de sus efectos en el sector salud. Económicas Cuc, 40(1), 27-46. https://doi.org/10.17981/econcuc.40.1.2019.02

Scatolon, E. (2014). Factura Electrónica En Los Sistemas De Informacion. Aplicación En Las Pymes Mendocinas. Universidad Nacional de Cuyo. Retrieved from http://bdigital.uncu.edu. ar/objetos_digitales/7052/42-scatolon-tesisfce. pdf 